\title{
Hour Of Sleep
}

National Cancer Institute

\section{Source}

National Cancer Institute. Hour Of Sleep. NCI Thesaurus. Code C64593.

Beginning hour of sleep. 